
\title{
28 Research Square \\ The Predictive and Diagnostic Potential of Symptoms for Glioblastoma Patient Survival
}

Oliver Mrowczynski ( $\nabla$ omrowczynski@pennstatehealth.psu.edu )

Penn State Milton S Hershey Medical Center https://orcid.org/0000-0002-3860-1309

Ae Lim Yang

Penn State Health Milton S Hershey Medical Center

Jason Liao

Penn State College of Medicine

Sara Langan

Penn State College of Medicine

Elias Rizk

Penn State Health Milton S Hershey Medical Center

\section{Clinical Study}

Keywords: Glioblastoma, glioma, symptoms, diagnosis, prognosis, survival

Posted Date: February 16th, 2021

DOI: https://doi.org/10.21203/rs.3.rs-200717/v1

License: (c) (1) This work is licensed under a Creative Commons Attribution 4.0 International License. Read Full License 


\section{Abstract}

\section{Purpose}

Glioblastoma is a devastating malignancy with a dismal survival rate and median survival time of 14 months. Currently, the biomarkers for glioblastoma are mostly molecular and include EGFRvIll, ATRX, PTEN, IDH1, MGMT, and others. These prognostic tumor biomarkers are obtained through a surgical biopsy and thus not easily attainable. Clinicians would benefit from a robust, non-invasive, and readily available indicator for early diagnosis and accurate prognostication for glioblastoma patients.

Methods

In this study, we assessed whether specific patient symptoms could provide early diagnosis of glioblastoma. Further, if any patient symptomatology would provide clinicians with the ability to prognosticate patient survival more accurately. We retrospectively reviewed the clinical data for 218 patients. We determined whether symptoms including headache, weakness, seizure, memory loss/confusion, visual changes, speech changes, and loss of consciousness led to a patient being diagnosed earlier and if any of these symptoms predicted a diminished patient survival.

\section{Results}

Our study determined that weakness and memory loss/confusion were symptoms that predicted diminished survival, and weakness alone was the symptom that predicted earlier diagnosis.

Conclusion: This study further elucidates the complexities of glioblastoma and provides clinicians with more data for their patients when discussing prognostication after diagnosis of glioblastoma.

\section{Introduction}

Glioblastoma is a malignant brain tumor in adults with a dismal survival rate and median survival time of fourteen months [1]. Glioblastoma unfortunately affects five per one hundred thousand people, making it the most common malignant brain tumor in adults [2]. Currently, the standard protocol for treatment, includes maximal safe surgical resection with adjuvant chemotherapy of temozolomide and radiation in large doses [3]. The current prognostic markers for glioblastoma are molecular and obtained through a surgical biopsy. These biomarkers include EGFRvIII, ATRX, PTEN, IDH1, MGMT, and others [1, 3-9]. Some are also currently used as markers to prognosticate patients and predict response to therapies [10,11]. A marker that is both readily attainable and acts as a robust prognostic and early diagnostic indicator would be beneficial for patients suffering from glioblastoma.

This study assesses whether symptoms including headache, weakness, seizure, memory loss/confusion, visual changes, speech changes, and loss of consciousness led to a patient being diagnosed earlier and if any of these symptoms predicted a diminished patient survival. The patient clinical exam is easily attainable, thus making it an optimal marker. Understanding the relationship between glioblastoma 
patient symptomatology and diagnosis and prognosis may pave the way to utilizing symptoms as a robust and quick factor for patient prognostication. It also provides the clinicians with more data to use when discussing with patients their prognosis to more accurately provide patients with information about their disease.

In this study, we retrospectively analyze glioblastoma patients treated at our institution, comparing the time of diagnosis and patient survival depending on their specific symptomatology. Specifically, this study is looking at the date of diagnosis to date of death for the survival analysis, and looking at the date of the first symptom of diagnosis for the diagnostic investigation. We further aim to further elucidate the complexities of this devastating disease. This study helps determine the underlying nuances of glioblastoma. It provides clinicians more data to speak to patients when discussing prognostication after diagnosis of glioblastoma.

\section{Methods}

\subsection{Patient Population}

A retrospective chart analysis that included 218 histopathology confirmed diagnosed glioblastoma patients at the Pennsylvania State University Department of Neurosurgery from 2006 until 2016 was included. The patients in this study were all pathologically confirmed to have WHO grade IV glioblastoma multiforme. Most patients included in the present analysis had the standard protocol for glioblastoma treatment, including maximal safe surgical resection, chemotherapy with temozolomide, and high dose $60 \mathrm{~Gy}$ radiation. Patients who had a previous low-grade glioma and any therapeutic intervention for a previous low-grade glioma were excluded from the study. Patients whose date of death could not be accurately determined were excluded.

\subsection{Data Collection and Statistical Analysis}

The dataset consists of 218 subjects. Days from the first symptom to death, days from the first symptom to diagnosis, and days from diagnosis to death are calculated for each subject. The dependence of these three time-to-event variables on various predictors is modeled using Cox's proportional hazards models. These predictors include sex, symptoms including headache, weakness, seizure, memory loss and confusion, visual changes, speech changes, loss of consciousness; and treatments including resection, radiation, and temodar. Additionally, days from the first symptom to diagnosis is also used as a potential predictor for days from diagnosis to death. Specifically, this study is looking at the date of diagnosis to date of death for the survival analysis, and looking at the date of the first symptom of diagnosis for the diagnostic analysis. All data in this study were subjected to statistical analysis. Our final Cox model for each time-to-event variable consists of only statistically significant predictors. The analysis was conducted using R 3.4 ( $R$ foundation for statistical computing). A p-value $<0.05$ was deemed statistically significant. This study received institutional review board approval, IRB \#5691. 


\section{Results}

\subsection{Patient Characteristics}

The characteristics of our 218 patients are shown in Table 1. Patients' median age was 64 years old with a range of five to 88 years old. In our cohort, 110 patients were male, comprising $51 \%$ of our sample population, while 108 patients were female, comprising $49 \%$ of our sample population. We also described the number of patients with specific symptoms; some patients had multiple presenting symptoms. Four patients were asymptomatic, comprising $1.8 \%$ of our sample population. Sixty-five patients had a headache comprising $29.8 \%$ of our sample population. Seventy-seven presented with weakness comprising $35.3 \%$ of our sample population. Twenty-one had a seizure comprising $9.6 \%$ of the population. Eighty-six had memory loss/confusion comprising $39.5 \%$ of our sample population. Fifteen patients had visual changes comprising $6.9 \%$ of our sample population. Fifty-five had speech changes comprising $25.2 \%$ of our sample population. Four had a loss of consciousness, comprising $1.8 \%$ of our sample population. We also showed the patients in our sample who were treated. One hundred and thirtyfive patients had a surgical resection, comprising $61.9 \%$ of our sample population. One hundred and forty-eight had radiation comprising $67.9 \%$ of our sample population, and one hundred fifty-two had temodar comprising $69.7 \%$ of our sample population.

\subsection{Symptom Prediction of Survival}

Patient symptom as a predictor of survival is shown in Table 2. The older the patient, the greater the decrease in survival $\left(p=3.17 \times 10^{\wedge}-5\right.$, hazard ratio (HR) 1.027 [1.014-1.039]). Patients presenting with weakness had decreased survival, which was statistically significant $(p=0.0063$; HR 1.543 [1.131-2.106]). Patients presenting with memory loss/confusion decreased survival, which trended towards statistical significance ( $p=0.0588 ;$ HR 1.345 [0.989-1.83]). All other symptoms, including headache, seizure, visual changes, speech changes, loss of consciousness, did not predict survival. As a control for our study, we evaluated the predictor of survival when patients received treatment with resection, radiation, and/or temodar. Patients that had a resection had an increased survival $(p=0.00085 ;$ HR 0.593 [0.436-0.806]). Patients who had radiation and temodar also had an increased survival $\left(\left(p=1.06 \times 10^{\wedge}-10 ; H R 0.338\right.\right.$ [0.243-0.47]) and ( $p=<2 \times 10^{\wedge}-16 ;$ HR 0.222 [0.157-0.314]), respectively). We also looked at the effects of resection and weakness. We show that not having a resection led to a lower survival probability (Figure $1 A, P=0.04)$. We also show that patients with weakness in the group of patients who did have a resection tended towards a shorter and decreased survival probability (Figure 1B, $\mathrm{P}=0.08$ ).

\subsection{Symptom Prediction of Diagnosis}

Patient symptoms as a predictor of early diagnosis are shown in Table 3. Age and sex did not have any predictor of an earlier diagnosis. Patients that presented with weakness as a symptom had a statistically significant increase in their speed of diagnosis ( $p=0.0152 ;$ HR 1.428 [1.071-1.904]). All other symptoms, including headache, seizure, memory loss/confusion, visual changes, speech changes, loss of consciousness, did not predict survival. 


\section{Discussion}

Glioblastoma patients have a devastating prognosis; and, non-invasive patient prognosis markers are important to help patients understand their disease and for the physician to more accurately predict a patient's survival. Patient's symptoms are one of the most easily attainable factors of a glioblastoma patient presentation. The possibility of using this data to prognosticate better a patient's clinical course is something to be desired. The better characterization of a glioblastoma patient regarding symptomatology also helps to understand this complex disease further. These data help the clinician be more accurate and personalized when discussing a patient's prognosis and disease course. To our knowledge, this is the first study analyzing and characterizing symptoms with regards to glioblastoma patient diagnosis and prognosis.

Our data included controls, e.g. the patient's age and treatment with surgical resection and adjuvant chemoradiation. As is known, older patients have diminished survival, as shown in our data. Additionally, surgical resection and treatment with adjuvant chemotherapy and radiation showed to have increased survival, which is also shown in our data. These factors were used to demonstrate our patient cohort following the known trends in the literature.Weakness may be identified as a symptom earlier than other symptoms due to its ability to be easily identified by the patient; and, may be reported sooner than other symptoms, leading to an earlier diagnosis. Further, weakness could lead family to urge the patient to seek medical attention and be evaluated with brain imaging.Symptoms like visual changes may not bring the patient to be evaluated sooner as the patient may be in denial of the occurring changes. These findings are different than the KPS scale as KPS is a functional impairment scale and not specific to weakness as a presentation. As far as weakness and memory loss/confusion being a predictor for decreased survival, this may be because these symptoms cause significant decreases in daily function. The patient is unable to care for themselves in the same way, which may lead to decreased patient survival overall. We show that in the group of patients who did have a resection, weakness was an independent predictor of poor outcome.

This study's limitations include its retrospective nature and that it only assesses the outcomes of patients in one institution. It also did not take into account a patient's financial status, which may have an impact on the availability of treatments and, thus, patient survival.

\section{Conclusions}

This study demonstrates that weakness and memory loss/confusion were symptoms which predicted diminished survival, and weakness alone was the symptom that predicted earlier diagnosis. This study further helps to elucidate the complexities of glioblastoma and provides clinicians with more data they are able to speak to patients about when discussing prognostication after diagnosis of glioblastoma. Further studies must be performed in larger cohorts in other institutions to confirm this finding further.

\section{Declarations}


Funding: N/A

Conflicts of interest/Competing interests: N/A

Availability of data and material: N/A

Code availability: N/A

Authors' contributions: N/A

\section{References}

1. Stupp R, Mason WP, van den Bent MJ, Weller M, Fisher B, Taphoorn MJB, et al: Radiotherapy plus Concomitant and Adjuvant Temozolomide for Glioblastoma. N Engl J Med352:987-996, 2005 Available: http://www.nejm.org/doi/abs/10.1056/NEJMoa043330. Accessed 20 July 2017

2. Wen PY, Kesari S: Malignant Gliomas in Adults. N Engl J Med359:492-507, 2008 Available: http://www.ncbi.nlm.nih.gov/pubmed/18669428. Accessed 20 July 2017

3. Stupp R, Hegi ME, Mason WP, van den Bent MJ, Taphoorn MJ, Janzer RC, et al: Effects of radiotherapy with concomitant and adjuvant temozolomide versus radiotherapy alone on survival in glioblastoma in a randomised phase III study: 5-year analysis of the EORTC-NCIC trial. Lancet Oncol10:459-466, 2009 Available: http://linkinghub.elsevier.com/retrieve/pii/S1470204509700257. Accessed 20 July 2017

4. Chaurasia A, Park S-H, Seo J-W, Park C-K: Immunohistochemical Analysis of ATRX, IDH1 and p53 in Glioblastoma and Their Correlations with Patient Survival. J Korean Med Sci31:1208, 2016 Available: http://www.ncbi.nlm.nih.gov/pubmed/27478330. Accessed 20 July 2017

5. Liu F, Hon GC, Villa GR, Turner KM, Ikegami S, Yang H, et al: EGFR Mutation Promotes Glioblastoma through Epigenome and Transcription Factor Network Remodeling. Mol Cell60:307-318, 2015 Available: http://www.ncbi.nlm.nih.gov/pubmed/26455392. Accessed 20 July 2017

6. Sturm D, Witt H, Hovestadt V, Khuong-Quang D-A, Jones DTW, Konermann C, et al: Hotspot Mutations in H3F3A and IDH1 Define Distinct Epigenetic and Biological Subgroups of Glioblastoma. Cancer Cell22:425-437, 2012 Available: http://www.ncbi.nlm.nih.gov/pubmed/23079654. Accessed 20 July 2017

7. Ueki K, Ono Y, Henson JW, Efird JT, von Deimling A, Louis DN: CDKN2/p16 or RB alterations occur in the majority of glioblastomas and are inversely correlated. Cancer Res56:150-3, 1996 Available: http://www.ncbi.nlm.nih.gov/pubmed/8548755. Accessed 20 July 2017

8. van den Bent MJ, Gao Y, Kerkhof M, Kros JM, Gorlia T, van Zwieten K, et al: Changes in the EGFR amplification and EGFRvIII expression between paired primary and recurrent glioblastomas. Neuro Oncol17:935-941, 2015 Available: http://www.ncbi.nlm.nih.gov/pubmed/25691693. Accessed 20 July 2017 
9. Xu J, Li Z, Wang J, Chen H, Fang J-Y: Combined PTEN Mutation and Protein Expression Associate with Overall and Disease-Free Survival of Glioblastoma Patients. Transl Oncol7:196-205.e1, 2014 Available: http://www.ncbi.nlm.nih.gov/pubmed/24721394. Accessed 20 July 2017

10. Kleihues P, Ohgaki H: Primary and secondary glioblastomas: from concept to clinical diagnosis. Neuro Oncol1:44-51, 1999 Available: http://www.ncbi.nlm.nih.gov/pubmed/11550301. Accessed 20 July 2017

11. Ohgaki $\mathrm{H}$, Kleihues P: The Definition of Primary and Secondary Glioblastoma. Clin Cancer Res19:764-772, 2013 Available: http://www.ncbi.nlm.nih.gov/pubmed/23209033. Accessed 20 July 2017

\section{Tables}

Table 1: Patient Characteristics $(n=218)$ 


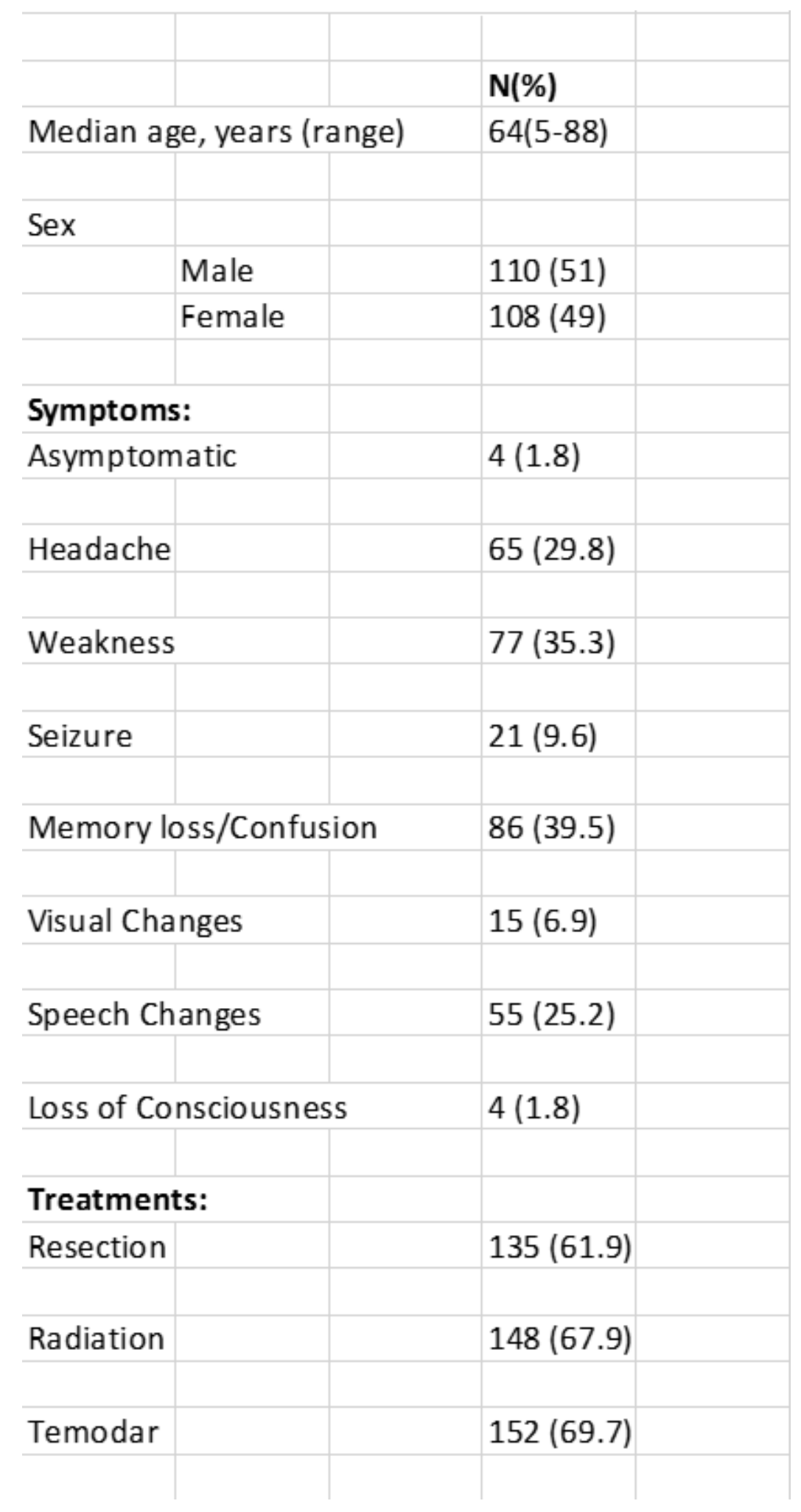

Table 2: Predictor of Survival 


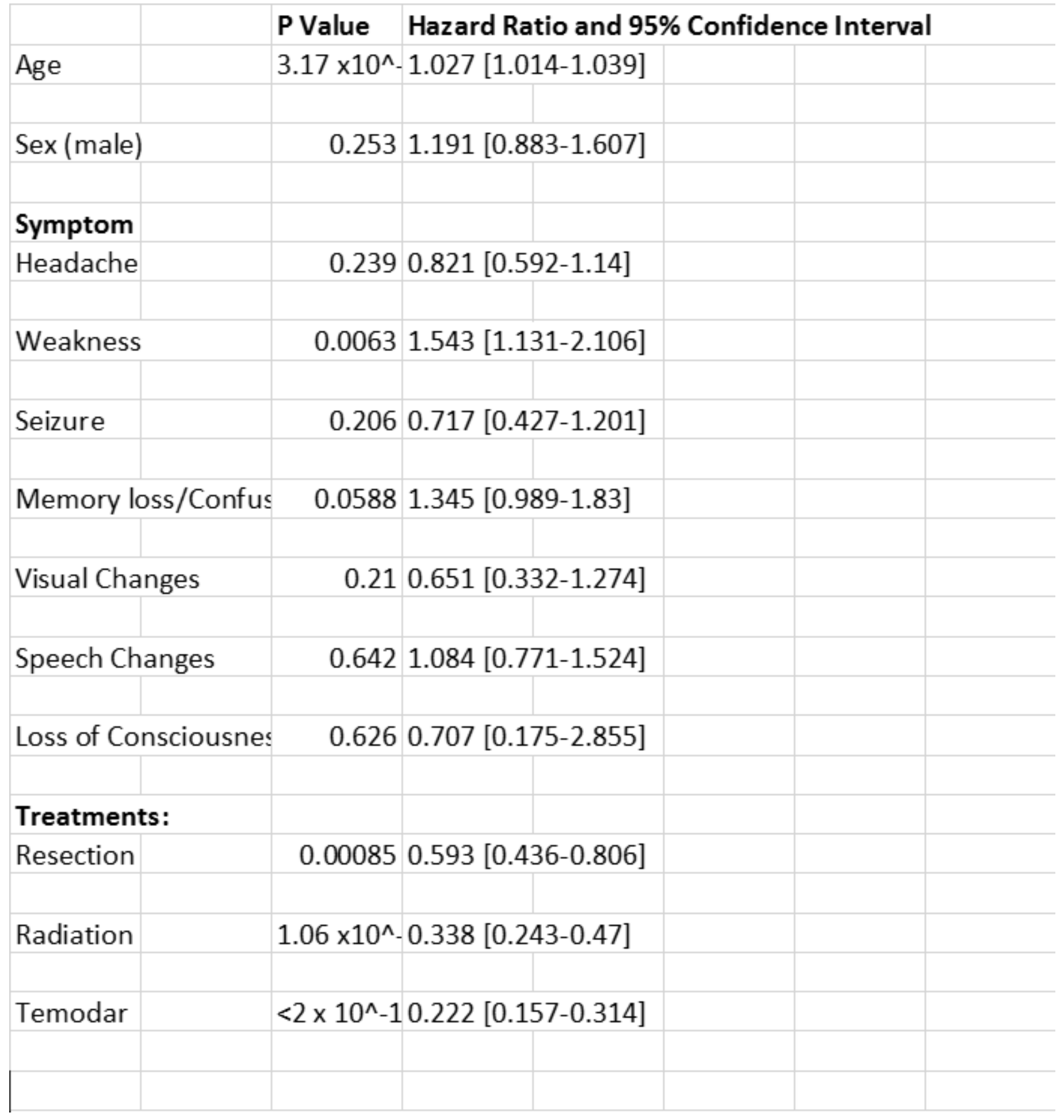

Table 3: Predictor of Diagnosis 


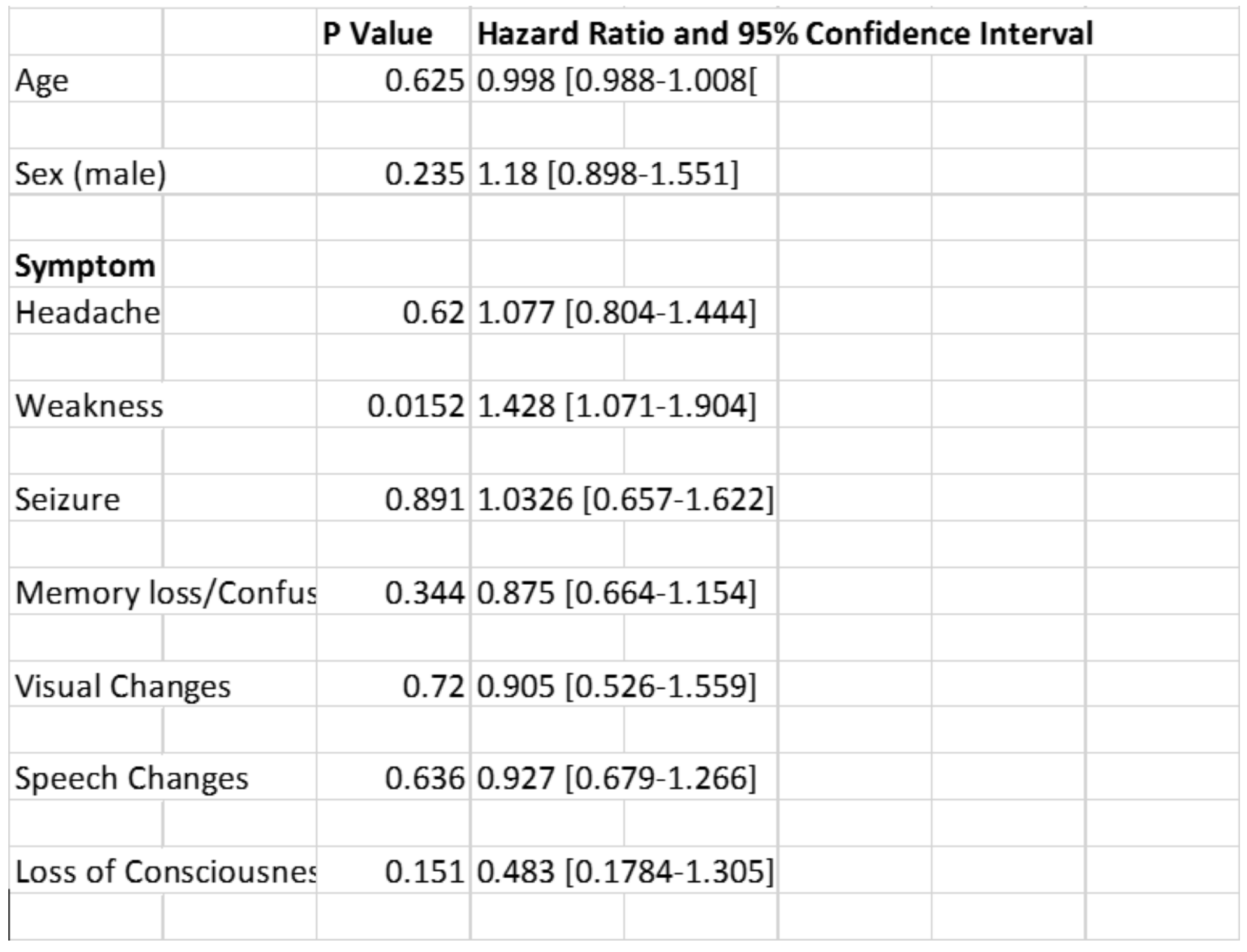

\section{Figures}



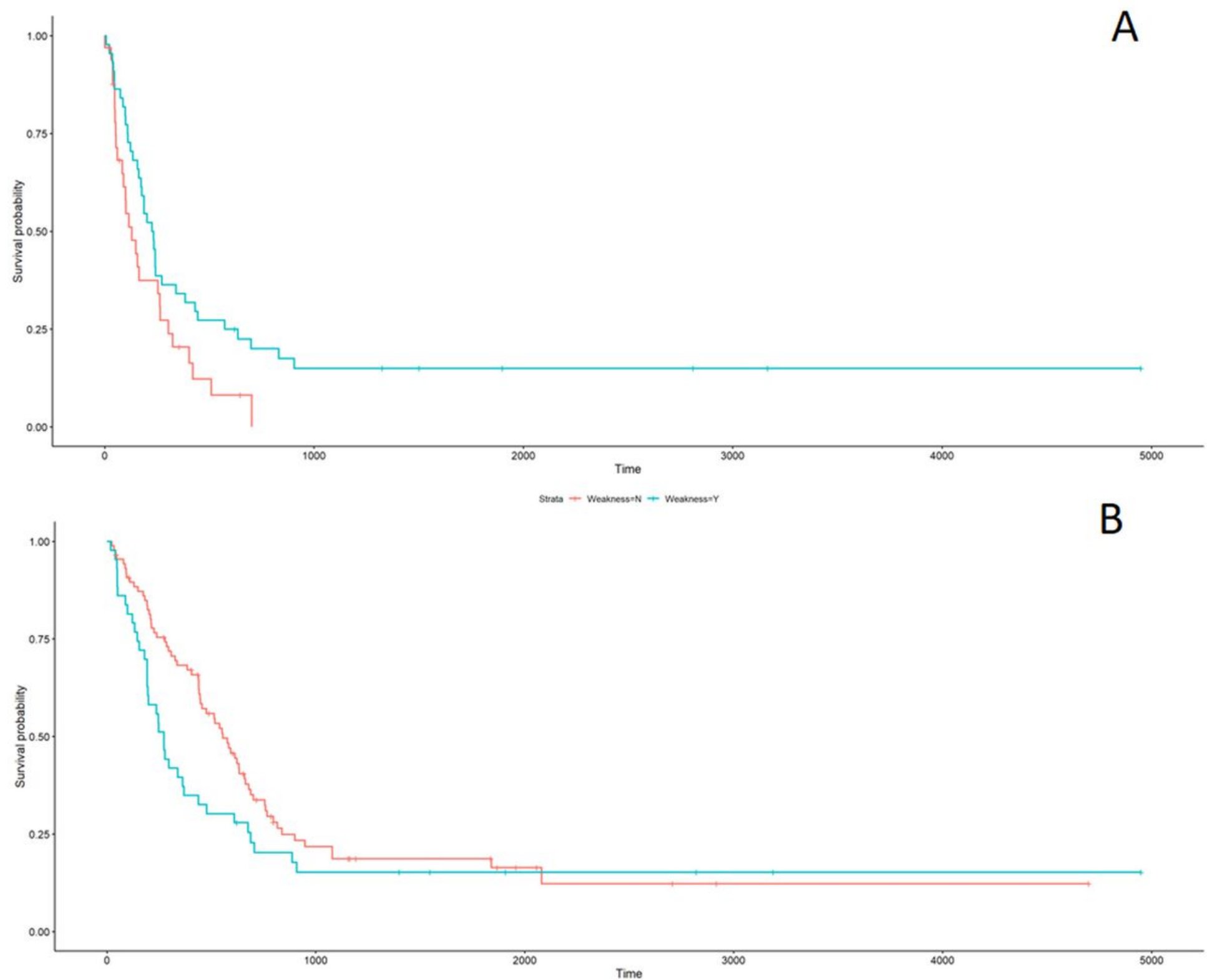

\section{Figure 1}

Effects of Weakness and Resection on Survival. (1A) The group of patients who had weakness, not having a resection led to a lower survival probability $(P=0.04)$. (1B) In the group of patients who did have a resection, patients with weakness trended towards a shorter a decreased survival probability (Figure 1B, $\mathrm{P}=0.08)$. 